\title{
Synthesis of Multivariable Nonlinear Controllers by Input/Output Linearization
}

This work concerns the synthesis of nonlinear controllers for multivariable nonlinear processes that make the closed-loop system linear in an input/output sense. Necessary and sufficient conditions for input/ output linearizability via static state feedback are derived as well as formulas for the feedback law. Once such a static state feedback is applied to the process, an external multivariable linear controller with integral action can control it to set point. The proposed control methodology is tested through simulations in a semibatch copolymerization reactor example.

\author{
Costas Kravaris \\ Masoud Soroush \\ Department of Chemical Engineering \\ University of Michigan \\ Ann Arbor, Ml 48109
}

\section{Introduction}

The nonlinear, strongly interacting nature of multivariable chemical processes necessitates the development of solid control methodologies that are capable of coping with both nonlinearities and interactions. However, in the process control field, the customary approach has been to neglect the nonlinearities by approximating the nonlinear model by a linear one and to apply linear theory to design linear controllers. In the case of processes with significant nonlinearities, the linear analysis is valid only in an infinitesimally small neighborhood of the operating point.

Recently, there has been considerable effort to design controllers so that the closed-loop response is exactly linear in a global sense. Input/output linearization involves finding nonlinear state feedback laws so that the input/output behavior of the closed-loop system is exactly linear. In the case of single-input/ single-output (SISO) systems, the problem is completely solved and explicit formulas for the input/output linearizing state feedback laws are available (Kravaris and Chung, 1987). Once such a state feedback law is applied to a nonlinear process, one can use an external linear controller with integral action for set point tracking and rejection of disturbances. The resulting control structure is called the Globally Linearizing Contol (GLC) structure (Kravaris and Chung, 1987).

In this paper, this methodology is extended to multi-input/ multi-output (MIMO) systems. In particular, we consider MIMO nonlinear systems with equal number of inputs and outputs of the form

$$
\left\{\begin{array}{l}
\dot{x}=f(x)+\sum_{j=1}^{m} g_{j}(x) u_{j} \\
y_{\mathrm{i}}=h_{i}(x), \quad i=1, \ldots, m
\end{array}\right.
$$

where

$$
u=\left[\begin{array}{c}
u_{1} \\
\vdots \\
u_{m}
\end{array}\right] \in \mathbb{R}^{m}, \quad y=\left[\begin{array}{c}
y_{1} \\
\vdots \\
y_{m}
\end{array}\right] \in \mathbb{R}^{m}, \quad x=\left[\begin{array}{c}
x_{1} \\
\vdots \\
x_{n}
\end{array}\right] \in \mathbb{R}^{n}
$$

$f(x)$ is a smooth vector field on $\mathrm{IR}^{n}, g_{1}(x), \ldots, g_{m}(x)$ are smooth vector fields on $\mathrm{IR}^{n}, h_{1}(x), \ldots, h_{m}(x)$ are smooth scalar fields on IR ${ }^{n}$ and $m \leq n$.

We start with a brief necessary review of the concepts of relative order and characteristic matrix in MIMO nonlinear systems. These concepts are used to derive necessary and sufficient conditions for existence of a state feedback that linearizes a MIMO nonlinear system in an input/output sense and also to provide explicit formulas for the control law. Stability issues arising in connection with input/output linearization are subsequently addressed. The special case where input/output decoupling is obtained together with input/output linearity is considered next. This is followed by a comparison of input/output linearization to linearization by immersion and to Volterra linearization. The theoretical results on input/output linearization lead to the Globally Linearizing Control (GLC) structure for the control of MIMO nonlinear systems. Finally, the GLC methodology is illustrated through a polymerization example. In this example, copolymer composition and number average molecular weight are controlled by manipulating the heat input and the rate of addition of one of the monomers in a semibatch reactor. Simulation results verify the theoretically-predicted linear closed-loop response and demonstrate the successful performance of the proposed control methodology. 


\section{Relative Orders and Characteristic Matrix}

This section reviews basic definitions that will be necessary for the development of our main results in the following section.

The concept of relative order of multivariable nonlinear systems has been introduced in the systems theory literature with a variety of names and in a variety of contexts [invertibility (Hirschorn, 1979), decoupling (Ha and Gilbert, 1986) and structure at infinity (Moog, 1988)].

Definition 1. Given a multivariable nonlinear system of the form of Eq. 1, we say that the $i$ th output $y_{i}$ has relative order $r_{i}$ if

$$
L_{\mathbf{g}_{j}} L_{j}^{k} h_{i}(x)=0, \quad j=1, \ldots, m, \quad k=0, \ldots, r_{i}-2
$$

and the row vector

$$
\begin{aligned}
& L_{g} L_{\mathrm{f}}^{\mathrm{r}_{\mathrm{i}}-1} h_{i}(x) \\
& \quad=\left[\begin{array}{lllll}
L_{g_{1}} L_{f}^{r_{i}-1} h_{i}(x) & L_{g_{2}} L_{f}^{r_{j}^{-1}} h_{i}(x) & \cdots & L_{g_{m}} L_{f}^{r_{j}-1} h_{i}(x)
\end{array}\right]
\end{aligned}
$$

is nonzero. Alternatively stated, $r_{i}$ is the smallest integer for which

$$
\begin{aligned}
& {\left[\begin{array}{llll}
L_{g_{1}} L_{f}^{r_{i}-1} h_{i}(x) & L_{g_{2}} L_{f}^{r_{i}-1} h_{i}(x) & \cdots & L_{g_{m}} L_{f}^{r_{i}-1} h_{i}(x)
\end{array}\right] } \\
& \neq\left[\begin{array}{lllll}
0 & 0 & \cdots & 0
\end{array}\right]
\end{aligned}
$$

Remark 1 . There may be singular points $P \in \mathbb{R}^{n}$ such that

$$
\begin{array}{rlll}
{\left[\begin{array}{llll}
L_{g_{1}} L_{j}^{r_{j}-1} h_{i}(x) & L_{g_{2}} L_{f}^{r_{i}-1} h_{i}(x) & \cdots & L_{g_{m}} L_{f}^{r_{i}-1} h_{i}(x)
\end{array}\right]_{\mid P}} & \\
& =\left[\begin{array}{lllll}
0 & 0 & \cdots & 0
\end{array}\right] .
\end{array}
$$

At these points, the relative orders $r_{i}$ are not defined. Singularities of this nature will not be considered here.

An immediate consequence of Definition 1 is the following formulas for the derivatives of the system outputs.

$$
\left\{\begin{array}{l}
\frac{d^{k} y_{i}}{d t^{k}}=L_{f}^{k} h_{i}(x), \quad k=1, \ldots, r_{i}-1 \\
\frac{d^{r_{i}} y_{i}}{d t^{r_{j}}}=L_{f}^{r_{j}} h_{i}(x)+\sum_{j=1}^{m} L_{g_{j}} L_{f}^{r_{j}-1} h_{i}(x) u_{j}
\end{array}\right.
$$

Therefore, $r_{i}$ is the smallest order of derivative of $y_{i}$ that explicitly depends on the vector $u$.

If a system output $y_{i}$ does not have a relative order, this means that $y_{i}$ and all its derivatives are not explicitly dependent on $u$; consequently, $y_{i}$ is not affected by $u$. In every well-formulated control problem, all outputs $y_{i}$ must possess a relative order. Otherwise, the system will not be output-controllable.

Relative orders depend only on the input/output behavior of the system as a result of the invariance of the quantities $L_{g_{j}} L_{f}^{k} h_{i}(x)$ under coordinate change. Consequently, in the case of linear systems, the $r_{i}$ 's will depend on the transfer function matrix $G(s)$ only and not on the particular state space realization. In particular, if $G(s)$ is in matrix fraction form

$$
G(s)=N(s)[D(s)]^{-1}
$$

where $N(s)$ and $D(s)$ are polynomial matrices and $D(s)$ is column reduced, then

$r_{i}=$ [Column Degree of the $i$ th Column of $\left.D(s)\right]$

- [Column Degree of the $i$ th Column of $N(s)]$

Definition 2 (Claude, 1986a). Consider a system of the form of Eq. 1 and assume that each output $y_{i}$ possesses a relative order $r_{i}$. The matrix

$$
C(x)=\left[\begin{array}{ccc}
L_{g_{1}} L_{f}^{r_{1}-1} h_{1}(x) & \cdots & L_{g_{m}} L_{f}^{r_{1}-1} h_{1}(x) \\
\vdots & & \vdots \\
L_{h_{1}} L_{f}^{r_{m}-1} h_{m}(x) & \cdots & L_{g_{m}} L_{f}^{r_{m}-1} h_{m}(x)
\end{array}\right]
$$

is called the characteristic matrix of the system of Eq. 1.

Remark 2. The characteristic matrix is also referred to as the decoupling matrix ( $\mathrm{Ha}$ and Gilbert, 1986) due to its significance to the nonlinear decoupling problem. We prefer the terminology characteristic matrix because $C(x)$ plays a much more fundamental role in multivariable control systems than just decoupling.

Remark 3. Because of the invariance of the quantities $L_{g_{i}} L_{j}^{k} h_{i}(x)$ under coordinate change, the characteristic matrix depends only on the input/output properties of the system.

\section{Input/Output Linearization}

This section contains our main theoretical results which extend the SISO input/output linearization method (Kravaris and Chung, 1987) to MIMO systems.

Definition 3. A multivariable nonlinear system of the form of Eq. 1 is called input/output linearizable if there exists a static state feedback of the form

$$
u=P(x)+Q(x) v
$$

with $Q(x)$ nonsingular and linear vector differential operators of the form

$$
\mathcal{L}_{p_{i}}=\sum_{k=0}^{\rho_{i}} \beta_{i k} \frac{d^{k}}{d t^{k}}, \quad i=1, \ldots, m
$$

with constant coefficients $\beta_{i k}=\left[\beta_{i k}^{1} \beta_{i k}^{2} \ldots \beta_{i k}^{m}\right]^{T} \in \mathbb{R}^{m}$ satisfying $\beta_{i p_{i}} \neq 0$ and

$$
\operatorname{det}\left[\left(\sum_{k=0}^{\rho_{1}} \beta_{1 k} s^{k}\right)\left(\sum_{k=0}^{\rho_{2}} \beta_{2 k} s^{k}\right) \cdots\left(\sum_{k=0}^{\rho_{m}} \beta_{m k} s^{k}\right)\right] \neq 0
$$

such that

$$
\sum_{i=1}^{m} \mathcal{L}_{\rho_{i}} y_{i}=v
$$

Remark 4. If a system gets input/output linearized by state feedback in the sense of the above definition, it may be convenient to think of the closed-loop system in the Laplace domain. From Eqs. 8 and 10 we immediately obtain the matrix fraction description of the closed-loop system:

$$
y(s)=[B(s)]^{-1} v(s)
$$


where

$$
B(s)=\left[\left(\sum_{k=0}^{p_{1}} \beta_{1 k} s^{k}\right)\left(\sum_{k=0}^{p_{2}} \beta_{2 k} s^{k}\right) \cdots\left(\sum_{k=0}^{\rho_{m}} \beta_{m k} s^{k}\right)\right]
$$

and $y(s)$ and $v(s)$ denote the Laplace transforms of $y(t)$ and $v(t)$, respectively. An immediate implication of Eq. 11 is that the requested closed-loop system does not have any finite zeros; this is in complete analogy with the SISO input/output linearization problem as formulated in Kravaris and Chung (1987). Also, Eqs. 11 and 12 provide a clear justification of the condition Eq. 9 in the definition of input/output linearizability: if Eq. 9 is not satisfied, the linear closed-loop system will be singular and this is definitely an undesirable situation.

Theorem 1 . A necessary condition for a system of the form of Eq. 1 to be input/output linearizable is that each output $y_{i}$ possesses a relative order. Furthermore, if $\rho_{l}$ are the orders of the linear operators in the closed-loop response (Eq. 10) and $r_{i}$ are the relative orders of the outputs of Eq. 1, then

$$
\rho_{i} \geq r_{i}, \quad i=1, \ldots, m
$$

The following theorem will provide a static-state feedback that makes the orders of the linear operators minimal, equal to $r_{i}$. We will see that, to be able to do that, we will need nonsingularity of the characteristic matrix. If this nonsingularity condition is violated, then a linearizing feedback will have to give orders of the linear operators in the closed-loop response larger than $r_{i}$.

Theorem 2. The following conditions are sufficient for a system of the form of Eq. 1 to be input/output linearizable:

i. Each output $y_{i}$ possesses a relative order $r_{i}$.

ii. Its characteristic matrix is nonsingular for all $\boldsymbol{x}$.

Furthermore, if the above conditions hold, then for any arbitrary $\beta_{i k} \in \mathbb{R}^{n}\left(k=0, \ldots, r_{i}\right.$ and $\left.i=1, \ldots, m\right)$ that satisfy Eq. 9 and

$$
\operatorname{det}\left[\beta_{1 r_{1}} \beta_{2 r_{2}} \ldots \beta_{m r_{m}}\right] \neq 0
$$

the state feedback

$$
\begin{aligned}
& u=\left\{\left[\beta_{1 r_{1}} \beta_{2 r_{2}} \cdots \beta_{m r_{m}}\right] L_{s}\right. {\left.\left[\begin{array}{c}
L_{f}^{r_{1}-1} h_{1}(x) \\
\vdots \\
L_{f}^{\prime-1} h_{m}(x)
\end{array}\right]\right\}^{-1} } \\
& \cdot\left\{v-\sum_{i=1}^{m} \sum_{k=0}^{r_{i}} \beta_{i k} L_{f}^{k} h_{i}(x)\right\}
\end{aligned}
$$

produces the closed-loop response

$$
\sum_{i=1}^{m} \sum_{k=0}^{r_{i}} \beta_{i k} \frac{d^{k} y_{i}}{d t^{k}}=v
$$

Remark 5 . The condition of Eq. 14 can be equivalently stated as follows: The matrix

$$
\left[\left(\sum_{k=0}^{r_{1}} \beta_{1 k} s^{k}\right)\left(\sum_{k=0}^{r_{2}} \beta_{2 k} s^{k}\right) \cdots\left(\sum_{k=0}^{r_{m}} \beta_{m k} s^{k}\right)\right]
$$

is column-reduced with column degrees $r_{1}, r_{2}, \ldots, r_{m}$, respectively. This implies that the determinantal degree of the matrix of Eq. 17 is equal to $r_{1}+r_{2}+\ldots+r_{m}$. Hence, the order of the closed-loop system is equal to $r_{1}+r_{2}+\ldots+r_{m}$.

Remark 6. The state feedback law of Eq. 15 is a direct generalization of the input/output linearizing state feedback law for SISO nonlinear systems (Kravaris and Chung, 1987):

$$
u=\frac{v-\sum_{k=0}^{r} \beta_{k} L_{f}^{k} h(x)}{\beta, L_{f}^{r-1} h(x)}
$$

Theorem 1 gives us a necessary condition for input/output linearizability: all outputs must have relative orders. Theorem 2 gives us a sufficient condition for input/output linearizability: it is enough that the system possesses relative orders and nonsingular characteristic matrix. To be able to characterize the class of input/output linearizable systems, we need conditions which are both necessary and sufficient.

In case that all outputs possess relative orders but the characteristic matrix is singular, it may be possible to modify the system by applying an invertible linear matrix differential operator to the system outputs, so that the modified system possesses a nonsingular characteristic matrix. One can then use an input/ output linearizing state feedback for the modified system to generate an input/output linearizing state feedback for the original system. These considerations lead to Theorem 3 which states the necessary and sufficient conditions for existence of an input/ output linearizing static feedback.

Theorem 3. A system of the form of Eq. 1 is input/output linearizable if and only if the following conditions are met

a. All the outputs have relative orders $r_{i}, i=1, \ldots, m$

b. There exists an invertible linear matrix differential operator $\mathcal{W}$ with constant coefficients such that

$$
\mathcal{W}\left[\begin{array}{c}
L_{f}^{r_{1}-1} h_{1}(x) \\
\vdots \\
L_{f}^{r_{m}-1} h_{m}(x)
\end{array}\right]
$$

is a function of $x$ only (independent of $u$ ) and the auxiliary system

$$
\left\{\begin{array}{c}
\dot{x}=f(x)+\sum_{j=1}^{m} g_{j}(x) u_{j} \\
y^{*}=\mathcal{W}\left[\begin{array}{c}
L_{f}^{r_{1}-1} h_{3}(x) \\
\vdots \\
L_{f}^{r_{m}-1} h_{m}(x)
\end{array}\right]
\end{array}\right.
$$

possess relative orders and a nonsingular characteristic matrix for all $x$.

Remark 7. The operator $\mathcal{W}$ is not, in general, unique. One could request, for example, the operator $\mathcal{W}$ to be a unimodular linear operator (i.e., its Laplace transform to be a unimodular matrix) or to provide relative orders $1,1, \ldots, 1$ to the auxiliary system of Eq. 19. 
Remark 8. If the system of Eq. I possesses a nonsingular characteristic matrix, then for every $\mathcal{W}=$ constant nonsingular $m \times m$ matrix (zero-order linear matrix differential operator), condition $(b)$ is automatically satisfied.

From a practical standpoint, the question is how such a $\mathcal{W}$ can be found and how can it be used to calculate a control law that induces linear input/output behavior. In this direction, one can use Hirschorn's inversion algorithm, since it tries to find a differential operator (in general state-dependent) such that, when applied to the outputs, it will provide a set of algebraic expressions in $x$ and $u$ that are solvable for $u$. If this operator turns out to have constant coefficients, it will automatically provide a $\mathcal{W}$. Hirschorn's algorithm will have to be started from

$$
\frac{d^{r_{i}-1} y_{i}}{d t^{r_{i}-1}}=L_{f}^{r_{i}-1} h_{i}(x)
$$

instead of the original outputs, in order to conform with the formulation of Theorem 3. For reasons of notational consistency and readability of the paper, Hirschorn's algorithm is outlined in Appendix A.

Theorem 4. If in Hirschorn's algorithm

$$
\left\{\begin{array}{l}
\rho^{\left(k^{*}\right)}=m \\
F_{\ell}(x)=\text { constant }, \quad \ell=0, \ldots, k^{*}-1
\end{array}\right.
$$

then the system Eq. 1 is input/output linearizable. Furthermore, given

$m \times 1$ matrices $\beta_{i k}, \quad i=0, \ldots, m, \quad k=0, \ldots, r_{i}-1$

$m \times\left(m-\rho^{(0)}\right), m \times\left(m-\rho^{(1)}\right), \ldots, m \times\left(m-\rho^{\left(k^{*}-1\right)}\right)$

matrices $\gamma_{0}, \gamma_{1}, \ldots, \gamma_{k^{*}-1}$ and an $m \times m$ invertible matrix $\Gamma$,

the state feedback

$$
\begin{aligned}
u=\left[\Gamma L_{g} H^{\left(k^{*}\right)}(x)\right]^{-1}\left\{v-\sum_{i=1}^{m} \sum_{k=0}^{r_{i}-1} \beta_{i k} L_{f}^{k} h_{i}(x)\right. \\
\left.-\sum_{\ell=0}^{k^{*}-1} \gamma_{\ell}\left[F_{\ell} \mid I_{m-\rho^{(\ell)}}\right] E_{\ell} L_{f} H^{(\ell)}(x)-\Gamma L_{f} H^{\left(k^{*}\right)}(x)\right\}
\end{aligned}
$$

produces the closed-loop response

$$
\begin{aligned}
\sum_{i=1}^{m} \sum_{k=0}^{r_{i}-1} \beta_{i k} \frac{d^{k} y_{i}}{d t^{k}}+\left\{\left[\sum_{\ell=0}^{k^{*}-1} \gamma_{\ell}\left[F_{\ell_{1}}^{\mid} I_{m-\rho^{(\ell)}}\right] E_{\ell} \mathcal{W}^{(\ell)}\right]\right. \\
\left.+\Gamma \mathcal{W}^{\left(k^{*}\right)}\right\}\left[\begin{array}{c}
\frac{d^{r_{1}} y_{1}}{d t^{r_{1}}} \\
\vdots \\
\vdots \\
\frac{d^{r_{m}} y_{m}}{d t^{r_{m}}}
\end{array}\right]=v
\end{aligned}
$$

where $\mathcal{W}^{(0)}, \mathcal{W}^{(1)}, \ldots, \mathcal{W}^{\left(k^{*}\right)}$ are linear matrix differential operators defined by

$$
\mathcal{W}^{(\ell+1)}=\left[\begin{array}{c:c}
I_{\rho^{(\ell)}} & 0 \\
\hdashline F_{\ell} \frac{d}{d t} & I_{m-\rho^{(\ell)}} \frac{d}{d t}
\end{array}\right] E_{\ell} \mathcal{W}^{(\ell)}, \quad \ell=0,1, \ldots, k^{*}-1
$$

\section{Stability of the Input/Output-Linearized System}

Consider first the case of a system of the form of Eq. 1 that possesses a nonsingular characteristic matrix: the state feedback of Eq. 15 will produce a linear input/output behavior governed by Eq. 16. The bounded input/bounded output (BIBO) stability characteristics of the $v-y$ system will then depend on the roots of the characteristic equation

$$
\operatorname{det}\left[\left(\sum_{k=0}^{r_{1}} \beta_{1 k} s^{k}\right)\left(\sum_{k=0}^{r_{2}} \beta_{2 k} s^{k}\right) \cdots\left(\sum_{k=0}^{r_{m}} \beta_{m k} s^{k}\right)\right]=0
$$

Since the parameters $\beta_{i k}$ are adjustable, they can always be chosen for closed-loop stability and fast dynamics.

In addition to input/output stability, it is important to obtain conditions for internal stability of the $v-y$ system, i.e., asymptotic stability of the states with respect to perturbations in the initial conditions under no external input $(v=0)$. When the system of Eq. 1 is subject to the state feedback of Eq. 15, the output dynamics of the unforced system is governed by

$$
\sum_{i=1}^{m} \sum_{k=0}^{r_{i}} \beta_{i k} \frac{d^{k} y_{i}}{d t^{k}}=0
$$

under appropriate initial conditions. Therefore, in view of $\mathrm{Re}$ mark 5 , by choosing the adjustable parameters $\beta_{i k}$ for BIBO stability, any initial conditions of the states will generate exponentially decaying signals for the outputs $y_{i}$ and their time derivatives $\left(\mathrm{d} y_{i}\right) /(\mathrm{dt}), \ldots,\left(\mathrm{d}^{\mathrm{r}_{\mathrm{i}}, y_{i}}\right) /\left(\mathrm{dt}^{\mathrm{r}_{i}-1}\right)$. Moreover, the outputs and their derivatives will get arbitrarily close to zero in finite time. Consequently, the asymptotic stability of the states (i.e., the stability as $t \rightarrow \infty$ ) of the unforced system will depend, for all practical purposes, on the asymptotic stability characteristics of the dynamical system resulting when

$$
y_{i}(t)=\frac{d y_{i}(t)}{d t}=\cdots=\frac{d^{r_{i}-1} y_{i}(t)}{d t^{r_{i}-1}}=0, \quad i=1, \ldots, m
$$

But this is exactly the zero dynamics of the system of Eq. 1 in the sense of Isidori and Moog (1988).

The foregoing considerations indicate that the input/output linearized system will be internally stable if the zero dynamics of the system of Eq. 1 (in the sense of Isidori and Moog) is stable. This is in complete analogy with the SISO results (Kravaris, 1988).

The case of an input/output linearizable system of the form of Eq. 1 with singular characteristic matrix can be treated similarly. The BIBO stability characteristics equation of the $v-y$ system will depend on the characteristic of Eq. 22. The internal stability characteristics will depend on the zero dynamics of the auxiliary system (Eq. 19) with $\mathcal{W}=\mathcal{W}^{\left(k^{*}\right)}$.

An in-depth treatment of the internal stability issue is postponed to a future communication; it will provide precise stability conditions on the zero dynamics that guarantee internal stability of the input/output linearized system.

\section{Input/Output Linearization vs. Input/Output Decoupling}

Freund (1975), Claude (1983b), and Ha and Gilbert (1986) considered the problem of finding a static state feedback such that, when applied to the system of Eq. 1, the closed-loop system 
becomes input/output decoupled, i.e., the $i$ th output depends on the $i$ th external input only:

$$
\left\{\begin{array}{c}
\sum_{k=0}^{\rho_{1}} \hat{\beta}_{1 k} \frac{d^{k} y_{1}}{d t^{k}}=v_{1} \\
\vdots \\
\sum_{k=0}^{\rho_{m}} \hat{\beta}_{m k} \frac{d^{k} y_{m}}{d t^{\hat{k}}}=v_{m}
\end{array}\right.
$$

for some $\hat{\beta}_{i k}$ scalar and $\rho_{i}$ positive integer. They showed:

(a) A system of the form of Eq. 1 is decouplable under static state feedback if, and only if, all its outputs possess relative orders and its characteristic matrix is nonsingular for all $x$.

(b) The required static state feedback for decoupled closedloop input/output behavior is given by

$$
\begin{aligned}
& u=\left[\begin{array}{ccc}
\hat{\beta}_{1 r_{1}} L_{g_{1}} L_{f}^{r_{1}-1} h_{1}(x) & \cdots & \hat{\beta}_{1 r_{1}} L_{g_{m}} L_{f}^{r_{1}-1} h_{1}(x) \\
\vdots & & \vdots \\
\hat{\beta}_{m r_{m}} L_{g_{1}} L_{f}^{r_{m}-1} h_{m}(x) & \cdots & \hat{\beta}_{m r_{m}} L_{g_{m}} L_{f}^{r_{m}-1} h_{m}(x)
\end{array}\right]^{-1} \\
& \left\{v-\left[\begin{array}{c}
\sum_{k=0}^{\prime} \hat{\beta}_{1 k} L_{f}^{k} h_{1}(x) \\
\vdots \\
\sum_{k=0}^{r_{m}} \hat{\beta}_{m k} L_{f}^{k} h_{m}(x)
\end{array}\right]\right\}
\end{aligned}
$$

where $r_{i}$ are the relative orders of the outputs $y_{i}$ and $\hat{\beta}_{i k}$ are scalar parameters. Clearly, the decoupled closed-loop system (Eq. 23) and the corresponding static state feedback (Eq. 24) are a special case of Eqs. 16 and 15, respectively, for

$$
\beta_{i k}=\hat{\beta}_{i k} e_{i}, \quad i=1, \ldots, m, \quad k=0, \ldots, r_{i}
$$

where

$$
e_{1}=\left[\begin{array}{l}
1 \\
0 \\
0 \\
\vdots \\
0 \\
0
\end{array}\right], \quad e_{2}=\left[\begin{array}{l}
0 \\
1 \\
0 \\
\vdots \\
0
\end{array}\right], \cdots, \quad e_{m}=\left[\begin{array}{c}
0 \\
0 \\
\vdots \\
0 \\
1
\end{array}\right]
$$

and, for satisying the condition of Eq. 14,

$$
\hat{\beta}_{i r_{i}} \neq 0, \quad i=1, \ldots, m
$$

Input/output linearization generalizes input/output decoupling in two important ways:

a) It allows obtaining an arbitrary linear input/output behavior. This is important because we know from linear systems that even though the design of a SISO linear controller is much easier than the design of a MIMO one, decoupled closed-loop response is not necessarily the best. In particular, in the case of ill-conditioned plants like high-purity distillation columns, decoupling is not a good choice (Skogestad et al., 1988). Disadvantages of decoupling arise from the fact that enforcement of structural constraints on the closed-loop dynamics (choosing $\beta_{i k}$ 's such that Eqs. 25 and 27 hold) may cause the closed-loop performance to deteriorate. Garcia and Morari (1985), in applying the internal model control (IMC) methodology to a hydrogen oxidation fixed-bed reactor model, have shown that it is impossible to obtain a perfectly decoupled response without loosing optimality of the closed-loop response. In addition to the above difficulties, a decoupling controller may demand larger values of the manipulated inputs in its attempt to modify the structural characteristics of the system.

b) The class of systems that are input/output-linearizable is significantly larger than the class of decouplable systems, with input/output linearizing state feedback.

- A decouplable nonlinear system is transformed into a decouplable (but not necessarily decoupled!) linear system.

- A nondecouplable nonlinear system is transformed into a nondecouplable linear system.

The control law (Eq. 15 or 21) allows controlling linear systems without having to impose any structural constraints on the closed-loop dynamics of the system. The control designer has, therefore, the flexibility to adjust the parameters $\beta_{i k}$ 's for fast closed-loop dynamics and desirable level of coupling.

\section{Comparison with Other Approaches for Linearizing the Input/Output Behavior}

In the previous sections we have formulated and solved the input/output linearization problem as a synthesis problem: given a postulated linear closed-loop input/output behavior depending on a number of adjustable parameters, we calculated the necessary feedback law. In this way, we obtained an explicit relationship between the control law and the achievable closedloop response.

On the other hand, recent work in the theoretical literature on Linearization by Immersion (Claude et al., 1983a; Claude, 1986b) and on Volterra Linearization (Isidori and Ruberti, 1984) formulated problems which are mathematically different from our synthesis problem but have a conceptual similarity: linear input/output behavior is sought in some sense.

In what follows we will provide a brief review of the above theoretical approaches and a comparison to our approach.

\section{Linearization by immersion}

Claude et al. (1983a) and Claude (1986b) used the abstract concept of immersion in order to mathematically define the situation where a system of the form of Eq. 1 has linear input/ output behavior. A nonlinear system of the form of Eq. 1 is said to be immersed into a linear system

$$
\left\{\begin{array}{l}
\dot{\zeta}=A \zeta+\sum_{j=1}^{m} b_{j} u_{j} \\
y_{i}=c_{i} \zeta, \quad i=1, \ldots, m
\end{array}\right.
$$

if there exists an analytic mapping $\mathcal{F}$ such that for every $x_{0} \in \mathbb{R}^{n}$, 
the system of Eq. 1 initialized at $x_{0}$, and the system of Eq. 28 initilized at $\mathfrak{F}\left(x_{0}\right)$ have the same generating series.

It is only an extremely restricted subclass of systems of the form of Eq. 1 that are immersable into a linear system. For this reason, one can try to find a static state feedback $u=P(x)+$ $Q(x) v$ with $Q(x)$ nonsingular so that the resulting closed-loop system is immersable into a linear system of the form of Eq. 28. This is the problem of linearization by immersion. Claude et al. (1983a) solved the problem of linearization by immersion under the following restriction on Eq. 28:

the space of unobservable states of Eq. 28 must be the same with the maximal $(A, B)$ invariant subspace contained in the intersection of $\operatorname{Kerc}_{i}, i=1, \cdots, m$.

They showed that the system of Eq. 1 can be immersed into Eq. 28 by the state feedback if, and only if, the feedback functions $P(x)$ and $Q(x)$ satisfy

a) For every $i=1, \cdots, m$,

$\left[L_{j}^{\prime-1} h_{f}(x)+L_{z} L_{j}^{\prime} h_{f}(x) P(x)\right] \in \mathbf{R}-\operatorname{span}\left\{L_{f}^{k} h_{i}(x)\right.$,

$$
\left.k=0, \cdots, r_{1}-1, i=1, \cdots, m\right\}
$$

b) $C(x) Q(x)$ - constant.

Note that an immediate consequence of the last condition and the nonsingularity of $Q(x)$ is that the system of Eq. 1 must have nonsingular characteristic matrix.

It is not difficult to show that the state feedback law of Eq. 15, which was derived under the assumption of nonsingular characteristic matrix, satisfies conditions $a$ and $b$ and that the resulting linear input/output system (Eq. 16) admits a realization of the form of Eq. 28 that satisfies the previously stated restriction.

In summary, although linearization by immersion appears to be a more general concept than the concept of input/output linearization, the imposed restriction on Eq. 28 limits it to systems with nonsingular characteristic matrix. In this case, it is possible to interpret the state feedback of Theorem 2 as providing a linearization by immersion.

\section{Volterra linearization}

Isidori and Ruberti (1984) used the Volterra series expansion in order to mathematically define the situation where a system of the form of Eq. 1 has linear input/output behavior. In particular, they requested the input-dependent part of the Volterra series expansion to be identical to the one of an autonomous linear system.

It is only an extremely restricted subclass of systems of the form of Eq. 1 that are linear in the above sense of Volterra series. For this reason, one can try to find a static state feedback $\boldsymbol{u}=$ $P(x)+Q(x) v$ with $Q(x)$ nonsingular so that the resulting closed-loop system is linear in the sense of Volterra series. This is the problem of Volterra linearization (Claude, 1986b). Isidori and Ruberti (1984) considered the following sequence of Toepliz matrices

$$
\theta_{k}(x)=\left[\begin{array}{cccc}
T_{0}(x) & T_{1}(x) & \cdots & T_{k}(x) \\
0 & T_{0}(x) & \cdots & T_{k-1}(x) \\
\vdots & \vdots & \vdots & \vdots \\
0 & 0 & \cdots & T_{0}(x)
\end{array}\right], k=0,1,2, \ldots
$$

where

$$
T_{k}(x)=L_{\varepsilon}\left[\begin{array}{c}
L_{f}^{k} h_{1}(x) \\
\vdots \\
L_{f}^{k} h_{m}(x)
\end{array}\right], \quad k=0,1,2, \ldots
$$

and denoted by $\sigma\left[\theta_{k}(x)\right]$ the rank of $\theta_{k}(x)$ for a fixed $x$ and by $\rho\left[\theta_{k}(x)\right]$ the rank of $\theta_{k}(x)$ as a matrix function [i.e., the number of $\mathbb{R}$-linearly independent rows of $\left.\theta_{k}(x)\right]$. They showed that a necessary and sufficient condition for the system of Eq. 1 to be Volterra linearizable under static state feedback is that

$$
\sigma\left[\theta_{k}(x)\right]=\rho\left[\theta_{k}(x)\right], \text { for all } k \geq 0
$$

To be able to check the above rank condition, Isidori and Ruberti (1984) suggested Silverman's structure algorithm for search and isolation of independent rows of each of the Toeplitz matrices $\theta_{k}(x)$. If the rank condition is met, the structure algorithm will also yield a set of equations for $P(x)$ and $Q(x)$ that provide one Volterra-linearizing state feedback.

The Volterra linearization concept is more general than the concept of input/output linearization we defined earlier, since the latter is restricted to a linear input-output behavior which is finite-dimensional, nonsingular, and without zeros. However, developing precise connections between the results of Theorem 3 and the Isidori-Ruberti rank condition is an open research problem.

Considering the particular state feedback generated by Silverman's algorithm in Isidori and Ruberti (1984), it is possible to show that it yields a linear finite-dimensional input-output behavior with no zeros. Consequently, it will belong to the class of state feedback laws of Theorem 4 if, and only if, it leads to a nonsingular closed-loop system.

\section{Globally Linearizing Controllers for Multivariable Nonlinear Systems}

We saw earlier that if an input/output-linearizable MIMO system of the form of Eq. 1 is subject to the state feedback

$$
u=\Psi(x, v)
$$

where

$$
\begin{aligned}
\Psi(x, v)=\left\{\left[\begin{array}{rr}
\beta_{1 r_{1}} & \beta_{2 r_{2}} \cdots \beta_{m r_{m}}
\end{array}\right] L_{g}\left[\begin{array}{c}
L_{f}^{r_{1}-1} h_{1}(x) \\
\vdots \\
L_{f}^{r_{m}-1} h_{m}(x)
\end{array}\right]\right\}^{-1} \\
\cdot\left\{v-\sum_{i=1}^{m} \sum_{k=0}^{r_{i}} \beta_{i k} L_{f}^{k} h_{i}(x)\right\}
\end{aligned}
$$


in case the sytem of Eq. I has a nonsingular characteristic matrix, and

$$
\begin{aligned}
\Psi(x, v)= & {\left[\Gamma L_{g} H^{\left(k^{*}\right)}(x)\right]^{-1}\left\{v-\sum_{i=1}^{m} \sum_{k=0}^{r_{i}} \beta_{i k} L_{f}^{k} h_{i}(x)\right.} \\
& \left.-\sum_{\ell=0}^{k^{*}-1} \gamma_{\ell}\left[F_{\ell} \mid I_{m-p^{(\ell)}}\right] E_{\ell} L_{f} H^{(\ell)}(x)-\Gamma L_{f} H^{\left(k^{*}\right)}(x)\right\}
\end{aligned}
$$

in case it does not, the resulting input/output behavior of the $v-y$ system is exactly linear, given by $\mathrm{Eq} .16$ or 22 , respectively.

Once such a state feedback is applied to a MIMO nonlinear process, the problem of controlling the outputs to set point reduces to a linear multivariable control problem. The latter can be solved by using the already mature linear multivariable control theory. This motivates the control structure of Figure 1, which we call the Multiinput/Multioutput Globally Linearizing Control (MIMO GLC) structure.

In the special case where input/output decoupling is meaningful and desirable, one can use

$$
\begin{aligned}
& \Psi(x, v)=\left[\begin{array}{ccc}
\hat{\beta}_{1 r_{1}} L_{g_{1}} L_{f}^{r_{1}^{-1}} h_{1}(x) & \cdots & \hat{\beta}_{1,1} L_{g_{m}} L_{f}^{r_{1}-1} h_{1}(x) \\
\vdots & & \vdots \\
\hat{\beta}_{m r_{m}} L_{g_{1}} L_{f}^{r_{f}-1} h_{m}(x) & \cdots & \hat{\beta}_{m r_{m}} L_{g_{m}} L_{f}^{r^{-1}} h_{m}(x)
\end{array}\right]^{-1} \\
& \cdot\left\{v-\left[\begin{array}{c}
\sum_{k=0}^{n_{1}} \hat{\beta}_{1 k} L_{f}^{k} h_{1}(x) \\
\vdots \\
\sum_{k=0}^{r_{m}} \hat{\beta}_{m k} L_{f}^{k} h_{m}(x)
\end{array}\right]\right\}
\end{aligned}
$$

In this case, the external multivariable linear controller will consist of SISO linear controllers, one for each input/output pair $\left(v_{i}-y_{i}\right)$. For example, one can use PI

$$
\begin{aligned}
v_{i}=\bar{\beta}_{i 0} y_{i}^{s p}(t)+ & K_{c_{i}}\left[y_{i}^{s p}(t)-y_{i}(t)\right]+ \\
& \frac{K_{c_{i}}}{\tau_{i_{i}}} \int_{0}^{t}\left[y_{i}^{s p}(t)-y_{j}(t)\right] d t, i=1, \cdots, m
\end{aligned}
$$

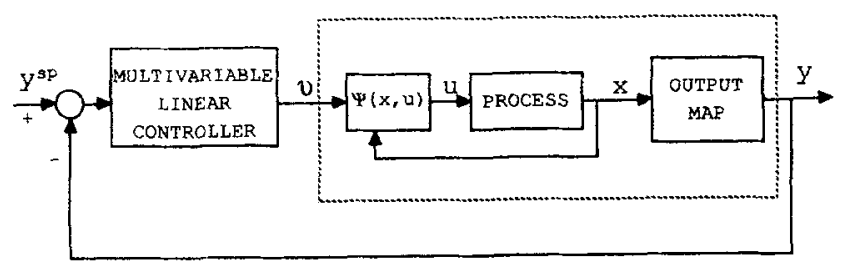

Figure 1. MIMO GLC structure. to obtain the overall closed-loop dynamics

$$
\begin{aligned}
\sum_{k=0}^{r_{i}} \hat{\beta}_{i k} \frac{d^{k} y_{i}}{d t^{k}}=\hat{\beta}_{i 0} y_{i}^{s p}(t) & +K_{c_{i}}\left[y_{i}^{s p}(t)-y_{i}(t)\right] \\
& +\frac{K_{c_{i}}}{\tau_{I_{i}}} \int_{0}^{t}\left[y_{i}^{s p}(t)-y_{i}(t)\right] d t, i=1, \cdots, m
\end{aligned}
$$

and the tuning of the controller parameters $K_{c_{i}}$ and $\tau_{t_{i}}$ becomes straightforward.

In summary, the MIMO GLC design procedure involves the following steps:

1. Compute the input/output linearizing state feedback.

2. Select the $\beta_{i k}$ 's so that the resulting linear $v-y$ system is BIBO stable, and has reasonably fast dynamics and desirable level of coupling.

3. Design an external linear multivariable controller for the $v-y$ system.

\section{Application to a Semibatch Copolymerization Reactor}

Tight control of polymer properties is one of the major problems in polymerization processes. In free radical copolymerization processes, controlling both copolymer composition and molecular weight is of primary importance. The control of composition and molecular weight in a free-radical solution copolymerization reactor by manipulating the heat input and monomer flow rate into the reactor is studied to illustrate the MIMO GLC methodology.

Diluted monomer $A$ and pure monomer $B$ are fed into the reactor, as shown in Figure 2, while the $\operatorname{sum} F_{T}$ of the two flow rates of the two streams is kept constant. The manipulated variables are the flow rate $F_{A}$ of the diluted monomer $A$ and the rate of heat exchange between the reactor and the jacket. Initially, the reactor is filled with the proper amount of monomers, initiator and solvent. In this example, the particular monomers are methyl methacrylate $(A)$ and vinyl acetate $(B)$, the initiator is azobisisobutyronitrile (AIBN) and the solvent is benzene.

Under the following assumptions:

- All the reactions are homogeneous.

- The reactor contents are perfectly mixed.

- Gel-effect is absent.

- The volume of the reacting mixture is changed due to the inflow of the monomers only.

- There is no polymer in the fluids entering the reactor.

The appropriate mass, mole and energy balances give a set of

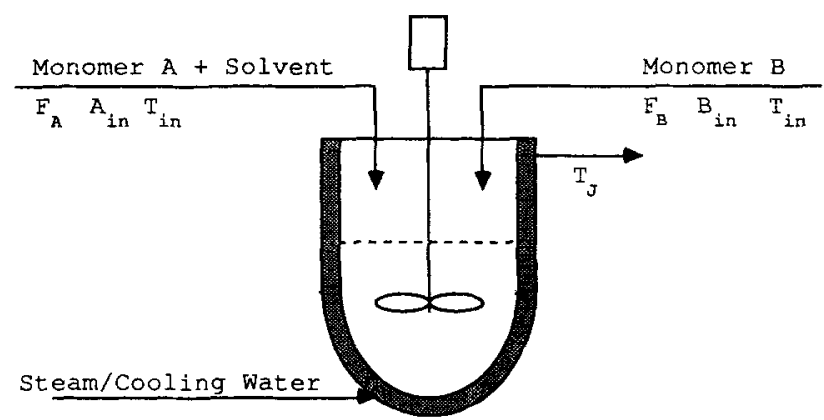

Figure 2. Semibatch reactor. 
ordinary differential equations (Ray et al., 1971b; Ray, 1972; Richards and Congalidis, 1987; Tsoukas et al., 1982), which can be represented in vector form as

$\frac{d}{d t}\left[\begin{array}{l}V \\ T \\ D_{0} \\ B \\ D_{1} \\ C_{A} \\ C_{B}\end{array}\right]=\left[\begin{array}{l}F_{T} \\ R_{A}(A, B, I, T)-\frac{A F_{T}}{V} \\ R_{B}(A, B, I, T)-\frac{\left(B-B_{i n}\right) F_{T}}{V} \\ R_{I}(I, T)-\frac{I F_{T}}{V} \\ R_{T}(A, B, I, T)-\frac{\left(T-T_{i n}\right) F_{T}}{V} \\ R_{D_{i}}(A, B, I, T)-\frac{D_{1} F_{T}}{V} \\ -R_{A}(A, B, I, T)-\frac{C_{A} F_{T}}{V} \\ -R_{B}(A, B, I, T)-\frac{C_{B} F_{T}}{V}\end{array}\right]$

$$
+\left[\begin{array}{l}
0 \\
0 \\
0 \\
0 \\
1 \\
0 \\
0 \\
0 \\
0
\end{array}\right] u_{1}+\left[\begin{array}{c}
0 \\
\frac{A_{\text {in }}}{V} \\
-B_{\text {in }} \\
V \\
0 \\
0 \\
0 \\
0 \\
0
\end{array}\right] u_{2}
$$

where the functions $R_{A}, R_{B}, R_{l}, R_{T}, R_{\mathrm{D}_{0}}$, and $R_{D_{1}}$ are known kinetic rate and heat of reaction expressions (see Appendix $\mathrm{C}$ for details). In the above equations, $u_{1}$ and $u_{2}$ are the manipulated inputs, the rate of heat exchange between the reactor, and the jacket $[U S(T,-T)] /(\rho C V)$ and the flow rate of the diluted monomer $A$ into the reactor $F_{A}$, respectively. The controlled outputs are $y_{1}=M_{n}$, the number average molecular weight and $y_{2}=Y_{A}$, the mole fraction of $A$-units in the dead copolymer, given by:

$$
y_{1}=\frac{D_{1}}{D_{0}}, \quad y_{2}=\frac{C_{A}}{C_{A}+C_{B}} .
$$

Equations 33 and 34 provide a state-space model of the polymerization reactor with $n=9$ states and $m=2$ inputs and outputs.
The model is clearly of the form of Eq. 1 with

$$
\begin{aligned}
& x^{T}=\left[\begin{array}{lllllll}
V A & B & I & T & D_{0} & D_{1} & C_{A} \\
C_{B}
\end{array}\right], \\
& f(x)=\left[\begin{array}{l}
f_{1}(x) \\
f_{2}(x) \\
f_{3}(x) \\
f_{4}(x) \\
f_{5}(x) \\
f_{6}(x) \\
f_{7}(x) \\
R_{A}\left(x_{2}, x_{3}, x_{4}, x_{5}\right)-\frac{x_{2} F_{T}}{x_{1}} \\
f_{8}(x) \\
R_{9}\left(x_{2}, x_{3}, x_{4}, x_{5}\right)-\frac{\left(x_{3}-B_{i n}\right) F_{T}}{x_{1}} \\
R_{I}\left(x_{4}, x_{5}\right)-\frac{x_{4} F_{T}}{x_{1}} \\
R_{T}\left(x_{2}, x_{3}, x_{4}, x_{5}\right)-\frac{\left(x_{5}-T_{i n}\right) F_{T}}{x_{1}} \\
R_{D_{0}}\left(x_{2}, x_{3}, x_{4}, x_{5}\right)-\frac{x_{6} F_{T}}{x_{1}} \\
R_{D_{1}}\left(x_{2}, x_{3}, x_{4}, x_{5}\right)-\frac{x_{9} F_{T}}{x_{1}} \\
-R_{A}\left(x_{2}, x_{3}, x_{4}, x_{5}\right)-\frac{x_{8} F_{T}}{x_{1}} \\
-R_{B}\left(x_{2}, x_{3}, x_{4}, x_{5}\right)-\frac{x_{9} F_{T}}{x_{1}}
\end{array}\right], \\
& g_{1}(x)=\left[\begin{array}{l}
0 \\
0 \\
0 \\
0 \\
1 \\
0 \\
0 \\
0 \\
0
\end{array}\right], \quad g_{2}(x)=\left[\begin{array}{c}
0 \\
\frac{A_{i n}}{x_{1}} \\
-B_{i n} \\
x_{1} \\
0 \\
0 \\
0 \\
0 \\
0 \\
0
\end{array}\right] \text {, } \\
& h_{1}(x)=\frac{x_{7}}{x_{6}}, \quad h_{2}(x)=\frac{x_{8}}{x_{8}+x_{9}} \text {. }
\end{aligned}
$$

We follow the procedure given previously to find the relative orders of this system and then apply MIMO GLC. Since

$$
\begin{aligned}
& L_{g_{1}} h_{1}(x)=0, \quad L_{g_{2}} h_{1}(x)=0, \quad L_{g_{1}} h_{2}(x)=0, \\
& L_{g_{2}} h_{2}(x)=0, \quad\left[L_{g_{1}} L_{f} h_{1}(x) \quad L_{g_{2}} L_{f} h_{1}(x) \neq\left[\begin{array}{ll}
0 & 0
\end{array}\right],\right. \\
& {\left[L_{g_{1}} L_{f} h_{2}(x) \quad L_{g_{2}} L_{f} h_{2}(x)\right] \neq\left[\begin{array}{ll}
0 & 0
\end{array}\right],}
\end{aligned}
$$

both outputs possess relative order 2 (i.e., $r_{1}=2, r_{2}=2$ ). Also, 
the characteristic matrix

$$
C(x)=\left[\begin{array}{ll}
L_{g_{1}} L_{f} h_{1}(x) & L_{g_{2}} L_{f} h_{1}(x) \\
L_{g_{1}} L_{f} h_{2}(x) & L_{g_{2}} L_{f} h_{2}(x)
\end{array}\right]
$$

$$
=\left[\begin{array}{ll}
\frac{\alpha_{x_{5}}}{x_{6}} & \frac{\left(\alpha_{x_{2}} A_{i n}-\alpha_{x_{3}} B_{i n}\right)}{x_{1} x_{6}} \\
\frac{\phi_{x_{5}}}{x_{8}+x_{9}} & \frac{\left(\phi_{x_{2}} A_{i n}-\phi_{x_{3}} B_{i n}\right)}{x_{1}\left(x_{8}+x_{9}\right)}
\end{array}\right]
$$

where

$$
\begin{array}{cl}
\alpha_{x_{i}}=\frac{\partial R_{D_{i}}}{\partial x_{i}}- & h_{1}(x) \frac{\partial R_{D_{0}}}{\partial x_{i}}, \\
\phi_{x}=\left(h_{2}(x)-1\right) \frac{\partial R_{A}}{\partial x_{i}}+h_{2}(x) \frac{\partial R_{B}}{\partial x_{i}}, & \\
\quad i=2,3,4,5
\end{array}
$$

is nonsingular. Therefore, Eq. 29 gives the static state feedback that makes the system linear in an input/output sense. In particular, for input/output-decoupled linear response, the necessary state feedback is given by Eq. 31 , i.e.

$$
\begin{aligned}
{\left[\begin{array}{l}
u_{1} \\
u_{2}
\end{array}\right]=} & \Psi(x, v)=\left[\begin{array}{ll}
\hat{\beta}_{12} L_{g_{1}} L_{f} h_{1}(x) & \hat{\beta}_{12} L_{g_{2}} L_{f} h_{1}(x) \\
\hat{\beta}_{22} L_{g_{1}} L_{f} h_{2}(x) & \hat{\beta}_{22} L_{g_{2}} L_{f} h_{2}(x)
\end{array}\right]^{-1} \\
& \cdot\left\{\left[\begin{array}{l}
v_{1} \\
\tau_{2}
\end{array}\right]-\left[\begin{array}{l}
\hat{\beta}_{10} h_{1}(x)+\hat{\beta}_{11} L_{f} h_{1}(x)+\hat{\beta}_{12} L_{f}^{2} h_{1}(x) \\
\hat{\beta}_{20} h_{2}(x)+\hat{\beta}_{21} L_{f} h_{2}(x)+\hat{\beta}_{22} L_{f}^{2} h_{2}(x)
\end{array}\right]\right\}
\end{aligned}
$$

where

$$
\begin{aligned}
L_{f} h_{1}(x) & =\frac{R_{D_{1}}-h_{1}(x) R_{D_{0}}}{x_{6}}, \\
L_{f} h_{2}(x) & =\frac{\left(h_{2}(x)-1\right) R_{A}+h_{2}(x) R_{B}}{x_{8}+x_{9}}, \\
L_{f}^{2} h_{1}(x) & =\frac{1}{x_{6}}\left[f_{2} \alpha_{x_{2}}+f_{3} \alpha_{x_{3}}+f_{4} \alpha_{x_{4}}+f_{5} \alpha_{x_{5}}-L_{f} h_{1}(x)\right. \\
L_{f}^{2} h_{2}(x) & =\frac{1}{x_{8}+x_{9}}\left[\left(2 R_{D_{0}}-\frac{x_{6} F_{T}}{x_{1}}\right)\right], \\
+L_{f} h_{2}(x)\left(2 \left(R_{A}+R_{x_{2}}+f_{3} \phi_{x_{3}}+f_{4} \phi_{x_{4}}+f_{5} \phi_{x_{5}}\right.\right. & \left.\left.\frac{\left(x_{8}+x_{9}\right) F_{T}}{x_{1}}\right)\right],
\end{aligned}
$$

and $\hat{\beta}_{i k}$ 's are scalar tunable parameters. The resulting $v-y$ system is described by

$$
\left\{\begin{array}{l}
\hat{\beta}_{10} y_{1}+\hat{\beta}_{11} \frac{d y_{1}}{d t}+\hat{\beta}_{12} \frac{d^{2} y_{1}}{d t^{2}}=v_{1} \\
\hat{\beta}_{20} y_{2}+\hat{\beta}_{21} \frac{d y_{2}}{d t}+\hat{\beta}_{22} \frac{d^{2} y_{2}}{d t^{2}}=v_{2}
\end{array}\right.
$$

In the external loop, two PI controllers (one for each output) are used, given by:

$$
\begin{aligned}
v_{1}=\hat{\beta}_{10} y_{1}^{s p}(t)+K_{c_{1}}\left(y_{1}^{s p}(t)\right. & \left.-y_{1}(t)\right) \\
& +\frac{K_{c_{1}}}{\tau_{l_{1}}} \int_{0}^{t}\left(y_{1}^{s p}(t)-y_{1}(t)\right) d t \\
v_{2}=\hat{\beta}_{20} y_{2}^{s p}(t)+K_{c_{2}}\left(y_{2}^{s p}(t)\right. & \left.-y_{2}(t)\right) \\
& +\frac{K_{c_{2}}}{\tau_{l_{2}}} \int_{0}^{t}\left(y_{2}^{s p}(t)-y_{2}(t)\right) d t
\end{aligned}
$$

where $y_{1}^{s p}$ and $y_{2}^{s p}$ are the set points.

\section{Table 1. Kinetic and Physical Parameters}

$$
\begin{array}{ll}
Z_{I} & -4.47 \times 10^{14} 1 / \mathrm{s} \\
Z_{C_{a b}} & =4.21 \times 10^{11} \mathrm{~m}^{3} / \mathrm{kmol} \cdot \mathrm{s} \\
Z_{C_{b b}} & =1.61 \times 10^{9} \mathrm{~m}^{3} / \mathrm{kmol} \cdot \mathrm{s} \\
Z_{P_{a a}} & =3.21 \times 10^{6} \mathrm{~m}^{3} / \mathrm{kmol} \cdot \mathrm{s} \\
Z_{P_{b b}} & =6.31 \times 10^{6} \mathrm{~m}^{3} / \mathrm{kmol} \cdot \mathrm{s} \\
Z_{P_{a b}} & =1.23 \times 10^{5} \mathrm{~m}^{3} / \mathrm{kmol} \cdot \mathrm{s} \\
Z_{P_{b b}} & =2.10 \times 10^{8} \mathrm{~m}^{3} / \mathrm{kmol} \cdot \mathrm{s} \\
Z_{f_{a a}} & =3.21 \times 10^{1} \mathrm{~m}^{3} / \mathrm{kmol} \cdot \mathrm{s} \\
Z_{f_{b b}} & =1.58 \times 10^{3} \mathrm{~m}^{3} / \mathrm{kmol} \cdot \mathrm{s} \\
Z_{f a b} & =1.23 \times 10^{0} \mathrm{~m}^{3} / \mathrm{kmol} \cdot \mathrm{s} \\
Z_{f_{b a}} & -5.26 \times 10^{4} \mathrm{~m}^{3} / \mathrm{kmol} \cdot \mathrm{s} \\
-\Delta H_{P_{a a}} & =5.78 \times 10^{4} \mathrm{~kJ} / \mathrm{kmol} \\
-\Delta H_{P_{b a}} & =5.78 \times 10^{4} \mathrm{~kJ} / \mathrm{kmol} \\
\rho & =8.80 \times 10^{2} \mathrm{~kg} / \mathrm{m}^{3} \\
U & =5.00 \times 10^{-2} \mathrm{~kJ} / \mathrm{m}^{2} \cdot \mathrm{s} \cdot \mathrm{K} \\
S & =1.00 \times 10^{-1} \mathrm{~m}{ }^{2} \\
R & =8.31 \times 10^{0} \mathrm{~kJ} / \mathrm{kmol} \cdot \mathrm{K} \\
A_{i n} & =2.00 \times 10^{0} \mathrm{kmol} / \mathrm{m}^{3} \\
B_{i n} & -1.08 \times 10^{1} \mathrm{kmol} / \mathrm{m}^{3}
\end{array}
$$

$$
\begin{array}{ll}
E_{I} & =1.27 \times 10^{5} \mathrm{~kJ} / \mathrm{mol} \\
E_{C_{a b}} & =2.69 \times 10^{4} \mathrm{~kJ} / \mathrm{kmol} \\
E_{C_{b b}} & =4.00 \times 10^{3} \mathrm{~kJ} / \mathrm{kmol} \\
E_{P_{a a}} & =2.42 \times 10^{4} \mathrm{~kJ} / \mathrm{kmol} \\
E_{P_{b b}} & =1.80 \times 10^{4} \mathrm{~kJ} / \mathrm{kmol} \\
E_{P_{a b}} & =2.42 \times 10^{4} \mathrm{~kJ} / \mathrm{kmol} \\
E_{P_{b a}} & =1.80 \times 10^{4} \mathrm{~kJ} / \mathrm{kmol} \\
E_{f_{a a}} & =2.42 \times 10^{4} \mathrm{~kJ} / \mathrm{kmol} \\
E_{f_{b b}} & =1.80 \times 10^{4} \mathrm{~kJ} / \mathrm{kmol} \\
E_{f_{a b}} & =2.42 \times 10^{4} \mathrm{~kJ} / \mathrm{kmol} \\
E_{f_{b a}} & =1.80 \times 10^{4} \mathrm{~kJ} / \mathrm{kmol} \\
-\Delta H_{P_{a b}} & =8.75 \times 10^{4} \mathrm{~kJ} / \mathrm{kmol} \\
-\Delta H_{P_{b b}} & =8.75 \times 10^{4} \mathrm{~kJ} / \mathrm{kmol} \\
C & =2.01 \times 10^{0} \mathrm{~kJ} / \mathrm{kg} \cdot \mathrm{K} \\
T_{i n} & =3.40 \times 10^{2} \mathrm{~K} \\
F_{T} & =1.00 \times 10^{-5} \mathrm{~m} 3 / \mathrm{s} \\
M_{A} & =1.00 \times 10^{2} \mathrm{~kg} / \mathrm{kmol} \\
M_{B} & =8.61 \times 10^{1} \mathrm{~kg} / \mathrm{kmol} \\
f & =1.00 \times 10^{0}
\end{array}
$$




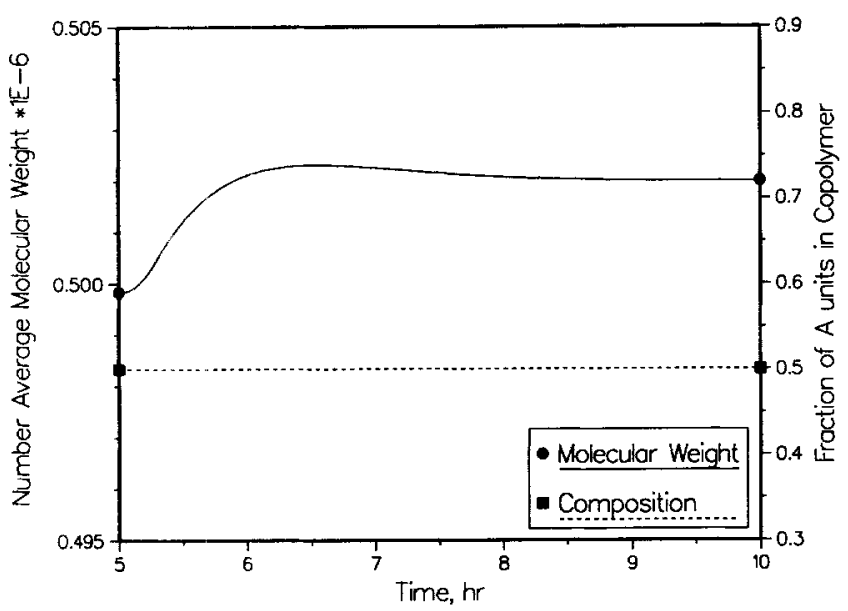

Figure 6. Variations of outputs resulting from the change in $y_{1}^{s p}$ introduced at $t=5.0 \mathrm{~h}$. (Continuation of Figure 3).

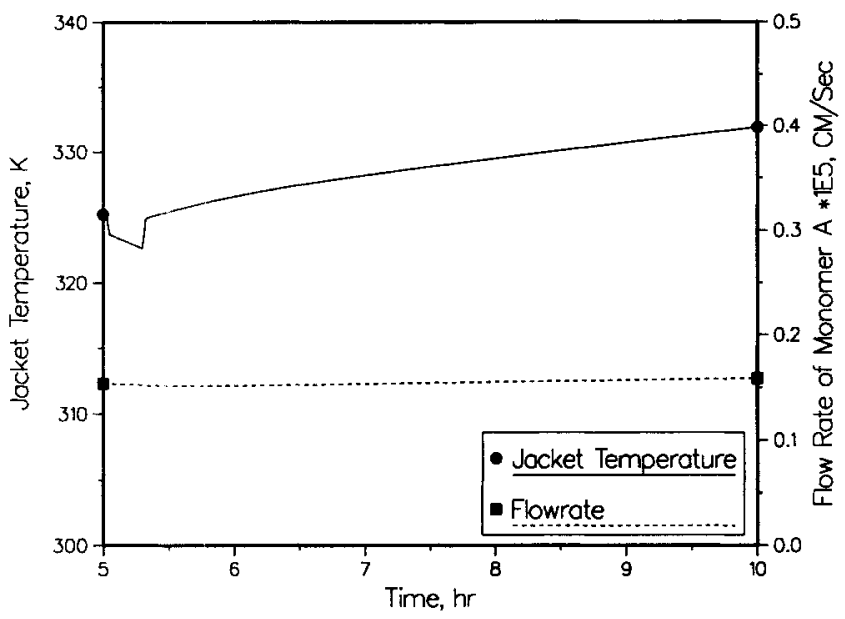

Figure 7. Variation of jacket temperature and flow rate of inlet monomer $\boldsymbol{A}$ stream, corresponding to Figure 6.

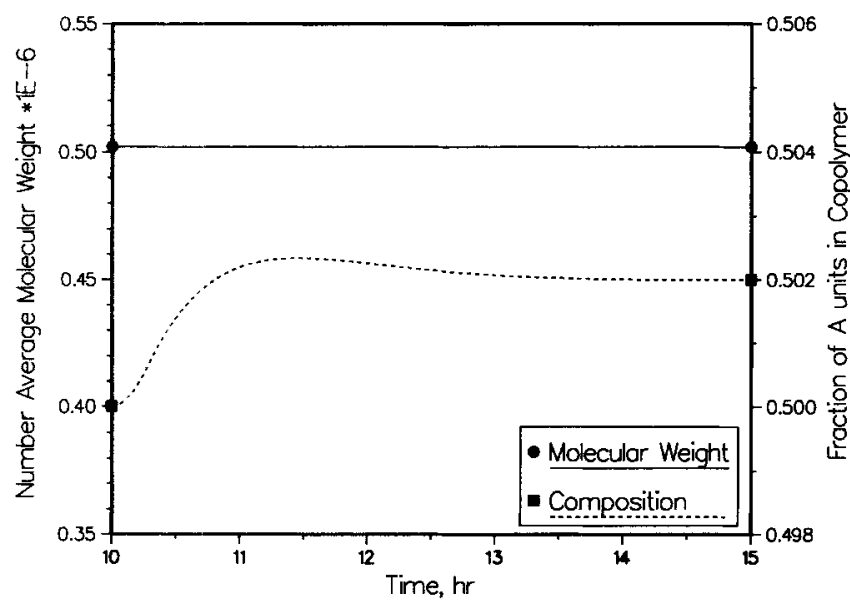

Figure 8. Variations of outputs resulting from the change in $y_{2}^{* D}$ introduced at $t=10.0 \mathrm{~h}$. (Continuation of Figure 6).

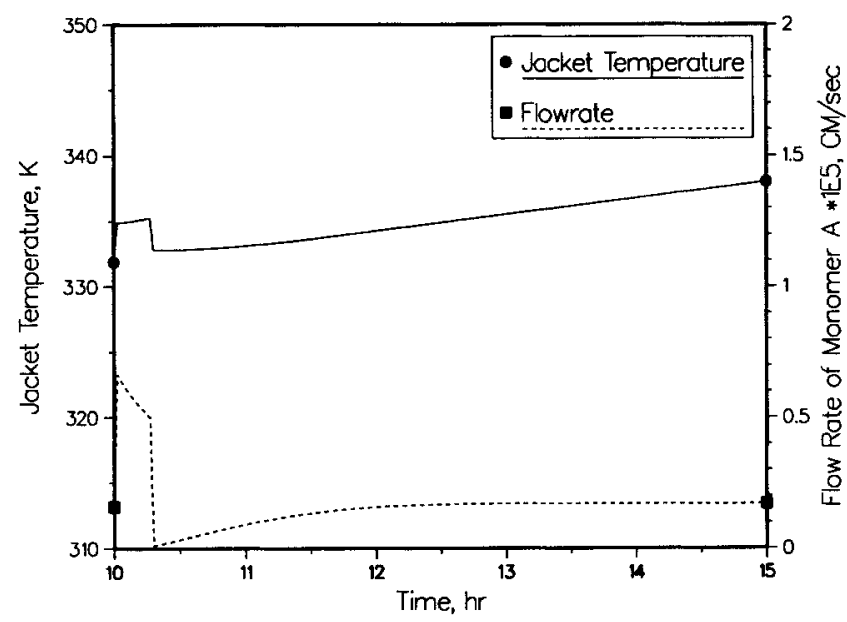

Figure 9. Variation of jacket temperature and flow rate of the diluted monomer $A$, corresponding to Figure 8.

strates that the system is indeed decoupled. Figures 7 and 9 depict the corresponding variations in $T_{J}$ and $F_{A}$.

It is interesting to note that the control system's action to achieve decoupling can be interpreted physically. A comparison of the values of the activation energies $E_{P_{a a}}, E_{P_{a b}}, E_{P_{b a}}, E_{P_{b b}}, E_{f_{a a}}$, $E_{f_{a b}}, E_{f_{b a}}$ and $E_{f_{b b}}$ for our copolymerization system shows that the instantaneous copolymer composition $\left(R_{A}\right) /\left(R_{A}+R_{B}\right)$ is almost independent of temperature. This independency can also be observed from Figures 6 and 7. We further observe from these figures that a demand for a higher number average molecular weight (a step change in $y_{l}^{s p}$ ) causes a decrease in jacket temperature. This agrees with the known fact that the lower temperature, the higher the number average molecular weight. Since this reduction in temperature has no significant effect on $Y_{A}$, no major change in $F_{A}$ is observed. Figure 9 shows the responses of $F_{A}$ and $T_{J}$ for a step change in the copolymer composition set point $y_{2}^{s p}$. This necessitates an increase in the flow rate of monomer $A$, which increases the concentration of monomer $A$ in the reactor. It can be deduced from a comparison of values of the rate constants $k_{P_{i j}}$ with $k_{f_{i}}$, that due to the increase in the concentration of monomer $A$, the increase in the rate of incorporation of monomer $A$ in the copolymer is larger than the rate of chain transfer to monomer reactions. This would tend to increase the number average molecular weight. But since the input/output linearized system is decoupled, $M_{n}$ should be unaffected. Consequently, the jacket temperature has to increase to compensate for the effect of increasing concentration of $A$.

Remark 10. The intuitive physical arguments of the previous paragraph indicate that the open-loop system is essentially oneway coupled: the second output $y_{2}=Y_{A}$ is affected only by $u_{2}=$ $F_{A}$ whereas the first output $y_{1}=M_{n}$ is affected by both manipulated inputs. Furthermore, the reactor temperature (which is directly affected by heat input $u_{1}$ ) has a much stronger effect on $y_{1}=M_{n}$ than the concentration of monomer $A$ (which is directly affected by $u_{2}=F_{A}$ ). In other words, the inherent coupling of the process is rather weak and this makes decoupling meaningful and feasible on intuitive grounds.

The effect of disturbances on the closed-loop response has also been studied. Simulations results indicated excellent distur- 
bance rejection ability of the control system when $F_{T}$ and $T_{\text {in }}$ undergo step or sinusoidal changes. Detailed description of the results is omitted for brevity.

\section{Notation}

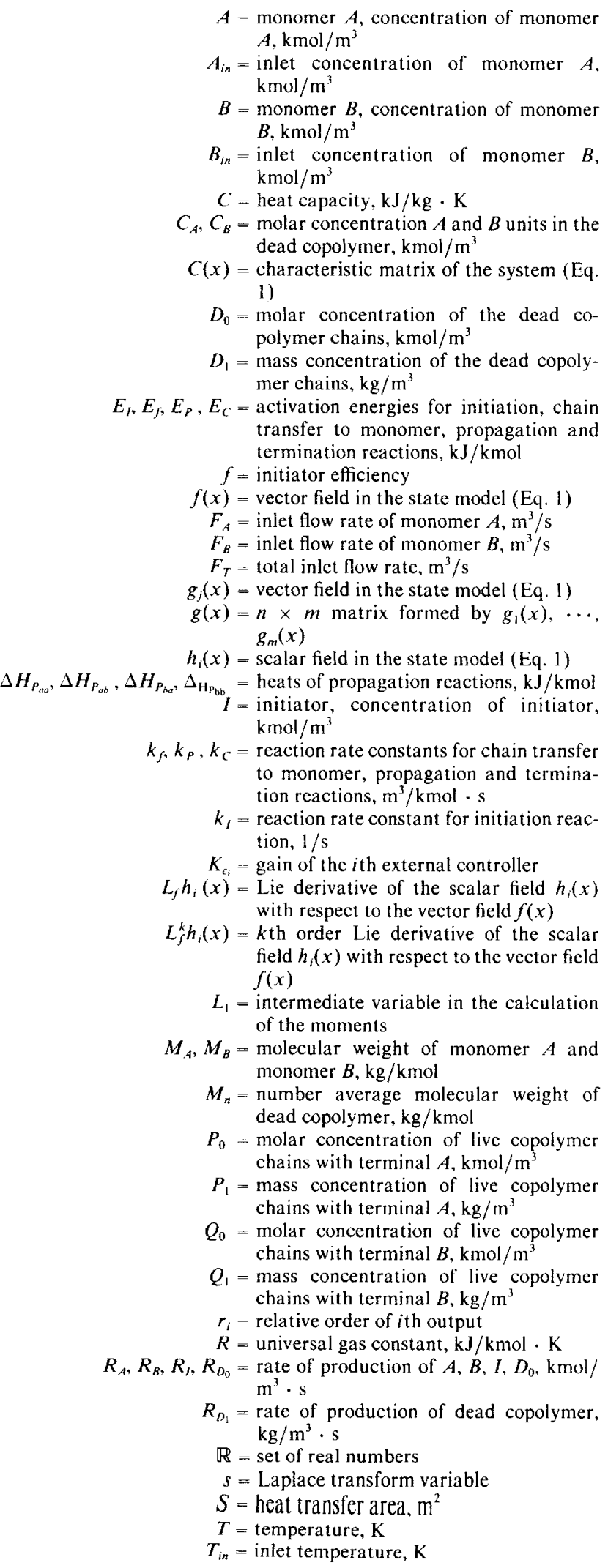

$T_{J}=$ jacket temperature, $\mathbf{K}$

$t=$ time, $\mathrm{s}$

$U=$ overall heat transfer coefficient, $\mathrm{kJ} / \mathrm{m}^{2}$. $\mathrm{s} \cdot \mathrm{K}$

$u=$ manipulated input vector

$u_{j}=j$ th manipulated input

$v=$ external input vector of the linear closedloop system

$V=$ volume of the reacting mixture, $\mathrm{m}^{3}$

$V_{a}, V_{b}-$ intermediate variables in the calculation of the moments

$x=$ vector of state variables

$Y_{A}=$ mole fraction of $A$ units in dead copolymer chain

$y=$ vector of output variables

$y_{i}=i$ th output variable

$y_{i}^{s p}=$ set point of $i$ th output variable

$Z_{f}, Z_{P}, Z_{C}=$ frequency factors for chain transfer to monomer, propagation and termination reactions, $\mathrm{m}^{3} / \mathrm{kmol} \cdot \mathrm{s}$

$Z_{I}=$ frequency factor for initiation reaction $1 / \mathrm{s}$

\section{Greek letters}

$\beta_{i j}=$ tunable parameters of the input/output linearized system

$\rho=$ density, $\mathrm{kg} / \mathrm{m}^{3}$

$\rho_{i}=$ orders of the linear operators defined in the closed-loop response (Eq. 10)

$\tau_{l_{i}}=$ integral time constant of the $i$ th external controller, $\mathrm{s}$

$\Psi=$ static state feedback in the MIMO GLC Structure

\section{Literature Cited}

Brandrup, J., and E. H. Immergut, eds., Polymer Handbook, 2nd ed., Wiley, New York (1975).

Claude, D., "Commande non Iteractive Simple des Systèmes non Linéaires par Bouclages Statiques Réguliers," C. R. Acad. Sc. Paris, 303, 833 (1986a)

-.-..., "Découplage des systèmes: du linéaire au non linéaire," Outils et Modeles Mathématiques pour l'Automatique, l'Analyse de Systèmes et le Traitement du Signal, I. D. Landau, ed., C.N.R.S., Paris, 533 (1983b).

"Everything You Always Wanted to Know about Linearization but Were Afraid to Ask," Algebraic and Geometric Methods in Nonlinear Control Theory. M. Fliess and M. Hazewinkel eds., D. Reidel Publishing Co., 181 (1986b).

Claude, D., M. Fliess, and A. Isidori, "Immersion, directe et par bouclage, d'um système non linéaire dans un linéaire," C.R. Acad. Sc Paris, 296, 237 (1983a).

Freund, E., "The Structure of decoupled Non-linear Systems," Int. J Contr., 21, 443 (1975).

Garcia, E. C., and M. Morari, "Internal Model Control. 3. Multivariable Control Law Computation and Tuning Guidelines," Ind. Eng. Chem., 24, 484 (1985).

Ha, I. J., and E. G. Gilbert, "A Complete Characterization of Decoupling Control Laws for a General Class of Nonlinear Systems," IEEE Trans. Autom. Contr., AC-31, 823 (1986).

Hirschorn, R. M., "Invertibility of Multivariable Nonlinear Control Systems," IEEE Trans. Autom. Contr., AC-24, 855 (1979).

Isidori, A., and C. H. Moog, "On the Nonlinear Equivalent of the Notion of Transmission Zeros," Modeling \& Adaptive Control, C. I. Byrnes and A. Kurzhanski, eds., Springer-Verlag, Berlin, 146 (1988)

Isidori, A., and A. Ruberti, "On the Synthesis of Linear Input-Output Responses for Nonlinear Systems," Sys. \& Contr. Lett., 4, 17 (1984).

Kravaris, C., "Input/Output Linearization: A Nonlinear Analog of Placing Poles at the Process Zeros," AIChE J., 34, 1803 (1988).

Kravaris, C., and C. B. Chung, "Nonlinear State Feedback Synthesis by Global Input/output Linearization," AIChE J., 33, 592 (1987). 
Moog, C. H., "Nonlinear Decoupling and Structure at Infinity," Mathematics of Control, Signals and Systems, 1, 257 (1988).

Ray, W. H., T. L. Douglas, and E. W. Godsalve, "Molecular Weight Distributions in Copolymer Systems. I. Living Copolymers," Macromol., 4(2), 162 (1971a)

Ray, W. H., T. L. Douglas, and E. W. Godsalve, "Molecular Weight Distributions in Copolymer Systems. II. Free Radical Copolymerization," Macromol., 4(2), 166 (1971b).

Ray, W. H., "On the Mathematical modeling of Polymerization Reactors," J. Macromol. Sci-Revs. Macromol. Chem., C8(1), 1 (1972).

Richard, J. R., and J. P. Congalidis, "Feedforward and Feedback Control of a Copolymerization Reactor," ACC Proc., 286 (1987).

Skogestad, S., M. Morari, and J. C. Doyle, "Robust Control of Ill-Conditioned Plants: High-Purity Distillation," IEEE Trans. Autom. Contr., 33, 1092 (1988).

Tsoukas, A., M. Tirrell, and G. Stephanopoulos, "Multiobjective Dynamic Optimization of Semibatch Copolymerization Reactors," Chem. Eng. Sci., 37(12), 1785 (1982).

\section{Appendix A: Hirschorn's Algorithm}

Set

$$
\begin{gathered}
H^{(0)}(x)=\left[\begin{array}{c}
L_{f}^{r_{1}-1} h_{1}(x) \\
\vdots \\
\vdots \\
L_{f}^{r^{-1}} h_{m}(x)
\end{array}\right], \\
\rho^{(0)}=\operatorname{Rank}\left[L_{g} H^{(0)}(x)\right] .
\end{gathered}
$$

Then, follow the sequence of steps, $k=0,1,2, \ldots$

Step $k$. Rearrange the rows of $L_{g} H^{(k)}(x)$ so that the first $\rho^{(k)}$ rows are linearly independent and denote by $E_{k}$ the corresponding elementary matrix that performs this row rearrangement. Find a $\left(m-\rho^{(k)}\right) \times \rho^{(k)}$ matrix $F_{k}(x)$ such that

$$
\left[F_{k}(x) I_{\left.m-\rho^{(k)}\right]}\right] E_{k} L_{g} H^{(k)}(x)=0
$$

where $I_{m-\rho^{(k)}}$ denotes the $\left(m-\rho^{(k)}\right) \times\left(m-\rho^{(k)}\right)$ identity matrix. Define

$$
\begin{aligned}
& H^{(k+1)}(x)=\left[\begin{array}{c}
\text { First } \rho^{(k)} \text { Rows of } E_{k} H^{(k)}(x) \\
\hdashline\left[F_{k}(x)\right. \\
\left.I_{m-\rho^{(k)}}\right] E_{k} L_{f} H^{(k)}(x)
\end{array}\right], \\
& \rho^{(k+1)}=\operatorname{Rank}\left[L_{g} H^{(k+1)}(x)\right] .
\end{aligned}
$$

Following the steps of the algorithm, a sequence of nonnegative integers $\rho^{(0)}, \rho^{(1)}, \rho^{(2)}, \ldots \rho^{(k)}, \ldots$ is produced such that

$$
0 \leq \rho^{(0)} \leq \rho^{(1)} \leq \rho^{(2)} \leq \cdots \leq \rho^{(k)} \leq \cdots \leq m .
$$

Thus, there is a least positive integer $k^{*}$ such that $\rho^{\left(k^{*}\right)}$ is maximal. In other words, the algorithm will always terminate after a finite number of steps, equal to $k^{*}$. At the last step, we will either have

$$
\rho^{\left(k^{*}\right)}=m,
$$

or none of the elements of

$$
\left[F_{k^{*}}(x) I_{m-\rho^{\left(k^{*}\right)}}\right] E_{k^{*}} L_{f} H^{\left(k^{*}\right)}(x)
$$

will possess a finite relative order.

\section{Appendix B: Proofs of Theorems}

Proof of Theorem 1. Suppose that some output $y_{i}$ does not have a relative order; this means that

$$
\frac{d^{k} y_{i}}{d t^{k}}=\text { independent of } u, \quad \text { for all } k
$$

Hence, after the state feedback $u=P(x)+Q(x) v$ is applied,

$$
\frac{d^{k} y_{i}}{d t^{k}}=\text { independent of } v, \quad \text { for all } k
$$

But then, the $i$ th row of the matrix

$$
\left[\left(\sum_{k=0}^{\rho_{1}} \beta_{l k} s^{k}\right)\left(\sum_{k=0}^{\rho_{2}} \beta_{2 k} s^{k}\right) \ldots\left(\sum_{k=0}^{\rho_{m}} \beta_{m k} s^{k}\right)\right]^{-1}
$$

must be identically zero. This leads to contradiction with Eq. 9 . The same argument can be used to show that if we assume that $\rho_{i}<r_{i}$ for some outputs, this also leads to contradiction.

Proof of Theorem 2. Consider a system of the form of Eq. 1 with relative orders $r_{1}, r_{2}, \cdots, r_{m}$. Then, using Eq. 5 for the time derivatives of the outputs $y_{i}$, we obtain

$$
\begin{aligned}
\sum_{i=1}^{m} \sum_{k=0}^{r_{i}} \beta_{i k} \frac{d^{k} y_{i}}{d t^{k}}= & \sum_{i=1}^{m} \sum_{k=0}^{r_{i}} \beta_{i k} L_{f}^{k} h_{i}(x) \\
& +\sum_{i=1}^{m} \beta_{i r_{i}}\left[\sum_{j=1}^{m} L_{g_{i}} L_{f}^{r_{i}-1} h_{i}(x) u_{j}\right] \\
= & \sum_{i=1}^{m} \sum_{k=0}^{r_{i}} \beta_{i k} L_{f}^{k} h_{i}(x)
\end{aligned}
$$

$$
+\left[\beta_{1 r_{1}} \beta_{2 r_{2}} \cdots \beta_{m r_{m}}\right] L_{g}\left[\begin{array}{c}
L_{f}^{r_{m}-1} h_{1}(x) \\
\vdots \\
L_{f}^{r_{m}-1} h_{m}(x)
\end{array}\right] u
$$

The nonsingularity of the characteristic matrix together with Eq. 14 guarantee that the matrix

$$
\left[\beta_{1 r_{1}} \beta_{2 r_{2}} \cdots \beta_{m r_{m}}\right] L_{g}\left[\begin{array}{c}
L_{f}^{r_{1}-1} h_{1}(x) \\
\vdots \\
L_{f}^{r_{m}-1} h_{m}(x)
\end{array}\right]
$$

is nonsingular. Thus, the state feedback (Eq. 15) is well-defined and will make the righthand side of Eq. B 1 equal to $v$. Hence, it will make the system input/output linear in the sense of Definition 3 and in particular it will produce the closed-loop response of Eq. 16.

Proof of Theorem 3. Necessity: Suppose that there exists a static state feedback

$$
u=P(x)+Q(x) v
$$


with $Q(x)$ nonsingular such that

$$
\sum_{i=1}^{m} \sum_{k=0}^{\beta_{i}} \beta_{i k} \frac{d^{k} y_{i}}{d t^{k}}=v
$$

Denoting by $r_{i}$ the relative orders of the outputs $y_{i}$ and defining the linear matrix differential operator

$$
\mathcal{W}=\left[\begin{array}{c}
\sum_{l=0}^{p_{1}-r_{1}} \beta_{1, r_{1+\ell}} \frac{d^{\prime}}{d t^{l}} \\
\vdots \\
\sum_{l=0}^{\rho_{m}-r_{m}} \beta_{m, r_{m, l}} \frac{d^{l}}{d t^{l}}
\end{array}\right]^{T}
$$

we can rewrite the closed-loop response as

$$
\sum_{i=1}^{m} \sum_{k=0}^{r_{i}-1} \beta_{i k} \frac{d^{k} y_{i}}{d t^{k}}+\frac{d}{d t}\left[\mathcal{W}\left[\begin{array}{c}
L_{f}^{r_{1}-1} h_{1}(x) \\
\vdots \\
L_{f}^{r_{m}-1} h_{m}(x)
\end{array}\right]\right]=v
$$

or, since $v=[Q(x)]^{-1}\{-P(x)+u\}$,

$$
\begin{aligned}
& \frac{d}{d t}\left[\mathcal{W}\left[\begin{array}{c}
L_{f}^{r_{1}-1} h_{1}(x) \\
\vdots \\
L_{f}^{r_{m}-1} h_{m}(x)
\end{array}\right]\right] \\
& =-\left\{\sum_{i=1}^{m} \sum_{k=0}^{r_{i}-1} \beta_{i k} L_{f}^{k} h_{i}(x)+[Q(x)]^{-1} P(x)\right\}+[Q(x)]^{-1} u .
\end{aligned}
$$

Thus, we conclude that

$$
\mathcal{W}\left[\begin{array}{c}
L_{f}^{r_{1}-1} h_{1}(x) \\
\vdots \\
L_{f}^{r_{m}-1} h_{m}(x)
\end{array}\right]
$$

is a vector function of $x$ only because otherwise its time derivative would depend on derivatives of $u$. Moreover, we will have

$$
L_{g}\left[\mathcal{W}\left[\begin{array}{c}
L_{f}^{r_{1}-1} h_{1}(x) \\
\vdots \\
L_{f}^{r_{m}-1} h_{m}(x)
\end{array}\right]\right]=[Q(x)]^{-1}=\text { nonsingular }
$$

Hence, the auxiliary system

$$
\left\{\begin{array}{c}
\dot{x}=f(x)+\sum_{j=1}^{m} g_{j}(x) u_{j} \\
y^{*}=\mathcal{W}\left[\begin{array}{c}
L_{f}^{r_{1}-1} h_{1}(x) \\
\vdots \\
L_{f}^{r_{m}-1} h_{m}(x)
\end{array}\right]
\end{array}\right.
$$

has relative orders $1,1, \ldots, 1$ and a nonsingular characteristic matrix.

Sufficiency: Set

$$
\mathcal{W}\left[\begin{array}{c}
L_{f}^{r_{1}-1} h_{1}(x) \\
\vdots \\
L_{f}^{r_{m}-1} h_{m}(x)
\end{array}\right]=\left[\begin{array}{c}
\lambda_{1}(x) \\
\vdots \\
\lambda_{m}(x)
\end{array}\right]
$$

and denote by $R_{1}, R_{2}, \cdots, R_{m}$ the relative orders of the auxiliary system

$$
\left\{\begin{array}{l}
\dot{x}=f(x)+\sum_{j-1}^{m} g_{j}(x) u_{j} \\
y_{i}^{*}=\lambda_{i}(x), \quad i=1, \ldots, m
\end{array}\right.
$$

Then from Theorem 2 the state feedback

$$
\begin{aligned}
& u=\left\{\left[\beta_{1 R_{1}} \beta_{2 R_{2}} \ldots \beta_{m R_{m}}\right] L_{g}\left[\begin{array}{c}
L_{f}^{R_{1}} \cdot \lambda_{1}(x) \\
\vdots \\
L_{f}^{R_{m}-1} \lambda_{m}(x)
\end{array}\right]\right\}^{-1} \\
& \cdot\left\{v-\sum_{i=1}^{m} \sum_{k=0}^{R_{i}} \beta_{i k} L_{f}^{k} \lambda_{i}(x)\right\}
\end{aligned}
$$

where $\left[\beta_{1 R_{1}} \beta_{2 R_{2}} \cdots \beta_{m R_{m}}\right]$ is nonsingular, will produce the closed-loop response

$$
\left[\left(\sum_{k=0}^{R_{1}} \beta_{1 k} \frac{d^{k}}{d t^{k}}\right)\left(\sum_{k=0}^{R_{2}} \beta_{2 k} \frac{d^{k}}{d t^{k}}\right) \cdots\left(\sum_{k=0}^{r_{m}} \beta_{m k} \frac{d^{k}}{d t^{k}}\right)\right]\left[\begin{array}{c}
y_{1}^{*} \\
\vdots \\
\vdots \\
y_{m}^{*}
\end{array}\right]=v
$$

or

$$
\left[\left(\sum_{k-0}^{R_{1}} \beta_{1 k} \frac{d^{k}}{d t^{k}}\right)\left(\sum_{k=0}^{R_{2}} \beta_{2 k} \frac{d^{k}}{d t^{k}}\right) \ldots\left(\sum_{k=0}^{R_{m}} \beta_{m k} \frac{d^{k}}{d t^{k}}\right)\right]
$$

$$
\mathcal{W}\left[\begin{array}{cccc}
\frac{d^{r_{1}-1}}{d t^{r_{1}-1}} & 0 & \cdots & 0 \\
0 & \frac{d^{r_{2}-1}}{d t^{r_{2}-1}} & \cdots & 0 \\
\vdots & \vdots & \cdots & \vdots \\
0 & 0 & \cdots & \frac{d^{r_{m}-1}}{d t^{r_{m}-1}}
\end{array}\right]\left[\begin{array}{c}
y_{1} \\
y_{2} \\
\vdots \\
y_{m}
\end{array}\right]=v
$$

Hence, it will linearize the system in an input/output sense.

Proof of Theorem 4. One can easily see by induction that

$$
\mathcal{W}^{(\ell)}\left[\begin{array}{c}
L_{f}^{r_{f}-1} h_{1}(x) \\
\vdots \\
L_{f}^{r_{m}-1} h_{m}(x)
\end{array}\right]=H^{(\ell)}(x), \quad \ell=0,1, \ldots, k^{*}
$$


On the other hand, since $\rho^{\left(k^{*}\right)}=m$,

$$
L_{g} H^{\left(k^{*}\right)}(x)=\text { nonsingular }
$$

Thus, setting $W^{\prime}=\mathcal{W}^{\left(k^{*}\right)}$, condition b of Theorem 3 is satisfied; the resulting auxiliary system has relative orders $1,1, \ldots, 1$ and nonsingular characteristic matrix. Hence, the system of Eq. 1 is input/output linearizable. Furthermore, one can also show by induction that

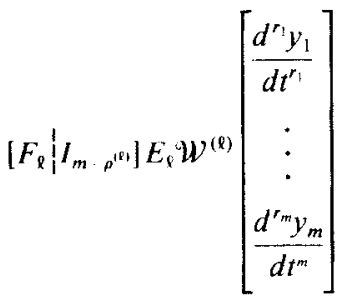

$$
\begin{aligned}
& =\left[F_{\ell} \mid I_{m-\rho^{(\ell)}}\right] E_{\ell} L_{f} H^{(\ell)}(x), \quad \ell=0, \ldots, k^{*}-1
\end{aligned}
$$

and

$$
\mathcal{W}^{\left(k^{*}\right)}\left[\begin{array}{c}
\frac{d^{r_{1}} y_{1}}{d t^{r_{1}}} \\
\vdots \\
\frac{d^{r_{m}} y_{m}}{d t^{m}}
\end{array}\right]=L_{f} H^{\left(k^{*}\right)}(x)+L_{g} H^{\left(k^{*}\right)}(x) u
$$

Thus,

$$
\begin{aligned}
& \sum_{i=1}^{m} \sum_{k=0}^{r_{1} \cdots i} \beta_{i k} \frac{d^{k} y_{i}}{d t^{k}}+\left\{\sum_{\ell=0}^{k^{*}-1} \gamma_{\ell}\left[F_{\ell} \mid I_{m-p^{(\ell)}}\right] E_{\ell} W^{(\ell)}+\Gamma \mathcal{W}^{\left(k^{*}\right)}\right\} \\
& {\left[\begin{array}{c}
\frac{d^{r_{1}} y_{1}}{d t^{r_{1}}} \\
\vdots \\
\cdot \\
\frac{d^{\prime} y_{m}}{d t^{m}}
\end{array}\right]=\sum_{i=1}^{m} \sum_{k=0}^{r_{i}-1} \beta_{i k} L_{f}^{k} h_{i}(x) } \\
&\left.+\sum_{\ell-0}^{k^{*}-1} \gamma_{\ell}\left|F_{\ell}\right| I_{m-p^{(\ell)}}\right] E_{\ell} L_{f} H^{(\ell)}(x)+\Gamma L_{f} H^{\left(k^{*}\right)}(x)+\Gamma L_{g} H^{\left(k^{*}\right)}(x) u
\end{aligned}
$$

The control law of Eq. 21 will make the righthand side of the above expression equal to $v$ and, therefore, will produce the closed-loop response of Eq. 22.

\section{Appendix C: Kinetic Mechanism and Rate Laws for Free Radical Copolymerization}

Making the following assumptions:

- Chain transfer to dead polymer does not occur.

- Inhibition, chain transfer to solvent, and termination by disproportionation reactions are neglected.

- The rate of reaction depends only on the free radical end group (independent of chain length).

- Quasisteady-state approximation and long chain hypothesis are used
- All reactions are elementary except for the initiation reaction.

And considering the standard reaction mechanism for homogeneous solution free-radical copolymerization (see steps a-d below), one can obtain the rates of consumption and/or production of each species (Ray et al., 1971; Ray, 1972; Tsoukas et al., 1982; Richards and Congalidis, 1987). In this mechanism, $P_{n, m}$, $Q_{n, m}$, and $D_{n, m}$ represent polymer chains containing $n$ units of monomer $A$ and $m$ units of monomer $B$. The letter $P$ denotes live polymer chains with terminal $A, Q$ live polymer chains with terminal $B$ and $D$ dead polymer chains.

a. Initiation Reactions

$$
\begin{array}{r}
I_{2} \stackrel{K_{I}}{\longrightarrow} 2 \dot{I} \\
I+A \stackrel{K_{l_{a}}}{\longrightarrow} P_{1,0} \\
I+B \stackrel{K_{l_{b}}}{\longrightarrow} Q_{0,1}
\end{array}
$$

\section{b. Propagation Reactions}

$$
\begin{aligned}
& P_{n, m}+A \stackrel{K_{P_{a a}}}{\longrightarrow} P_{n+1, m} \\
& P_{n, m}+B \stackrel{K_{P_{a b}}}{\longrightarrow} Q_{n, m+1} \\
& Q_{n, m}+A \stackrel{K_{P_{b a}}}{\longrightarrow} P_{n+1, m} \\
& Q_{n, m}+B \stackrel{K_{P_{b b}}}{\longrightarrow} Q_{n, m+1}
\end{aligned}
$$

\section{c. Termination by Coupling Reactions}

$$
\begin{gathered}
P_{n, m}+P_{r, q} \stackrel{K_{C_{a a}}}{\longrightarrow} D_{n+r, m+q} \\
P_{n, m}+Q_{r, q} \stackrel{K_{C_{a b}}}{\longrightarrow} D_{n+r, m+q} \\
Q_{n, m}+Q_{r, q} \stackrel{K_{C_{b b}}}{\longrightarrow} D_{n+r, m+q}
\end{gathered}
$$

d. Chain Transfer to Monomer Reactions

$$
\begin{aligned}
& P_{n, m}+A \stackrel{K_{f a a}}{\longrightarrow} D_{n, m+} P_{1,0} \\
& P_{n, m}+B \stackrel{K_{f a b}}{\longrightarrow} D_{n, m+} Q_{0,1} \\
& Q_{n, m}+A \stackrel{K_{f b a}}{\longrightarrow} D_{n, m+} P_{1,0} \\
& Q_{n, m}+B \stackrel{K_{f b b}}{\longrightarrow} D_{n, m+} Q_{0,1}
\end{aligned}
$$




\section{Reaction rate expressions}

$$
\begin{aligned}
R_{A}= & -A\left[\left(K_{P_{a a}}+K_{f_{a a}}\right) P_{0}+\left(K_{P_{b a}}+K_{f_{b a}}\right) Q_{0}\right] \\
R_{B}= & -B\left[\left(K_{P_{b b}}+K_{f_{b b}}\right) Q_{0}+\left(K_{P_{a b}}+K_{f_{b b}}\right) P_{0}\right] \\
R_{I}= & -K_{I} I \\
R_{T}= & \left(-\Delta H_{P_{a a}}\right) K_{P_{a a}} P_{0} A+\left(-\Delta H_{P_{a b}}\right) K_{P_{a b}} P_{0} B \\
& +\left(-\Delta H_{P_{b a}}\right) K_{P_{b a}} Q_{0} A+\left(-\Delta H_{P_{b h}}\right) K_{P_{b b}} Q_{0} B
\end{aligned}
$$

Applying the method of generating functions described by Ray et al. (1971a), Ray et al. (197lb), Ray (1972), and Tsoukas et al. (1982), we can calculate the molecular weight distribution of the free radical and dead copolymer molecules. Thus, the rate of production of zeroth and first moments of the molecular weight distribution of the dead copolymer (molar and mass concentration, respectively) are given by

$$
\begin{aligned}
R_{D_{0}}= & 0.5 K_{C_{a b}} P_{0}^{2}+K_{C_{a b}} P_{0} Q_{0}+0.5 K_{C_{b b}} Q_{0}^{2} \\
& +\left(K_{f_{c a}}+K_{f_{a b}}\right) P_{0}+\left(K_{f_{b b}}+K_{f_{b a}}\right) Q_{0} \\
R_{D_{1}}= & K_{C_{a a}} P_{0} P_{1}+K_{C_{a b}}\left(P_{0} Q_{1}+P_{1} Q_{0}\right)+K_{C_{b b}} Q_{0} Q_{1} \\
& +\left(K_{f_{a a}}+K_{f_{a b}}\right) P_{1}+\left(K_{f_{b b}}+K_{f_{h a}}\right) Q_{1}
\end{aligned}
$$

where

$$
\begin{aligned}
k_{i}= & Z_{i} \exp \left(\frac{-E_{i}}{R T}\right), \\
& i=I, P_{a a}, P_{a b}, P_{b a}, P_{b b}, C_{a a}, C_{b b}, f_{a a}, f_{a b}, f_{b a}, f_{b b} \\
k_{C_{a b}=} & \sqrt{k_{C_{a a}} k_{C_{b b}},} \\
L_{1}= & \frac{B\left(K_{P_{a b}}+K_{f_{b}}\right)}{A\left(K_{P_{b a}}+K_{f_{b a}}\right)}, \\
P_{0}= & \left(\frac{2 f K_{1} I}{K_{C_{a a}}+2 L_{1} K_{C_{a b}}+K_{C_{b b}} L_{1}^{2}}\right)^{0.5}, \\
Q_{0}= & L_{1} P_{0}, \\
V_{a}= & \left(K_{P_{a a}}+K_{f_{a a}}\right) A+\left(K_{P_{a b}}+K_{f_{a b}}\right) B+K_{C_{a b}} P_{0}+K_{C_{a b}} Q_{0}, \\
V_{b}= & \left(K_{P_{b b}}+K_{f_{b b}}\right) B+\left(K_{P_{b a}}+K_{f_{b a}}\right) A+K_{C_{b b}} Q_{0}+K_{C_{a b}} P_{0}, \\
P_{1}= & \frac{-R_{A} M_{A}\left(V_{b}-K_{P_{b b}} B\right)-K_{P_{b a}} A R_{B} M_{B}}{\left(V_{a}-K_{P_{a a}} A\right)\left(V_{b}-K_{P_{b b}} B\right)-K_{P_{b a}} K_{f a b} A B}, \\
Q_{1}= & \frac{-R_{B} M_{B}\left(V_{a}-K_{P_{a a}} A\right)-K_{P_{a b}} B R_{A} M_{A}}{\left(V_{a}-K_{P_{a a}} A\right)\left(V_{b}-K_{P_{b b}} B\right)-K_{P_{b o}} K_{f a b} A B} .
\end{aligned}
$$

Manuscript received Apr. 18. 1989, and revision received Nov. 27, 1989. 